\title{
Remarks on the Space-Time Behavior of Scattering Solutions to the Schrödinger Equations
}

By

\author{
Tohru OzawA*
}

\begin{abstract}
We consider the space-time behavior of scattering solutions to the Schrödinger equation

$$
\begin{aligned}
& i \partial_{t} u=H u \quad \text { on } \quad(x, t) \in \mathbb{R}^{n} \times \boldsymbol{R} \quad(n \geqq 1), \\
& u(x, 0)=\phi(x), \quad x \in \mathbb{R}^{n},
\end{aligned}
$$

where $H$ denotes a self-adjoint operator in the Hilbert space $\mathscr{H}:=L^{2}\left(\boldsymbol{R}^{n}\right)$. We prove the fact that every scattering solution which has the estimate

$$
\left|\left(e^{-i t H} \phi\right)(x)\right| \leqq C|t|^{-\alpha}(1+|x|)^{-\beta} \quad\left(|t| \geqq 1, x \in \mathbb{R}^{n}\right)
$$

for some $\alpha, \beta \in \mathbb{R}$ with $\alpha+\beta>n / 2$ vanishes identically.

Furthermore, we show, every non-trivial scattering solution has the estimate

where

$$
\liminf _{R \rightarrow+\infty} R^{-1} \int_{\Gamma_{R}^{\underline{T}}}\left|\left(e^{-i t H} \phi\right)(x)\right|^{2} d x d t>0,
$$

and

$$
\Gamma_{R}^{+}=\left\{(x, t) \in \mathbb{R}^{n} \times \boldsymbol{R} ; R<\left(|t|^{2}+|x|^{2}\right)^{1 / 2}<2 R, t>0\right\}
$$

$$
\Gamma_{\bar{R}}^{-}=\left\{(x, t) \in \boldsymbol{R}^{n} \times \boldsymbol{R} ; R<\left(|t|^{2}+|x|^{2}\right)^{1 / 2}<2 R, t<0\right\} .
$$
\end{abstract}

\section{$\S 0$. Introduction and Main Results}

In this article we consider the space-time behavior of scattering soultions to the Schrödinger equation

$$
\begin{aligned}
& i \partial_{t} u=H u \quad \text { on } \quad(x, t) \in \mathbb{R}^{n} \times \mathbb{R} \quad(n \geqq 1), \\
& u(x, 0)=\phi(x), \quad x \in \mathbb{R}^{n},
\end{aligned}
$$

where $H$ is a self-adjoint operator in the Hilbert space $\mathscr{H}=L^{2}\left(\boldsymbol{R}^{n}\right)$. A scatter-

Communicated by S. Matsuura, September 24, 1986.

* Research Institute for Mathematical Sciences, Kyoto University, Kyoto 606, Japan. 
ing solution means a solution of the form $e^{-i t H} \phi$ with $\phi \in \mathscr{H}_{\text {cont }}=\mathscr{A}_{\text {cont }}(H)$ (the continuous spectral subspace of $H$ ).

In the special case where $H$ takes the form

$$
H=H_{0}+V \text {, }
$$

where $H_{0}$ is the self-adjoint realization of $-\Delta$ in $\mathscr{H}$ and

$$
\left(1+|x|^{2}\right)^{s / 2} V \in L^{\infty}\left(\boldsymbol{R}^{n}\right) \quad \text { with } \quad s>1,
$$

the following theorem is well-known (see [8], [9] and [12]):

If $e^{-i t H} \phi$ has the estimate, for some $\varepsilon>0$ and for every compact set $K \subset R^{r}$.

$$
\left\|e^{-i t H} \phi\right\|_{L^{2}(K)} \leqq C_{K, \varepsilon} e^{-\varepsilon t}, \quad t \geqq 0,
$$

then $\phi$ vanishes identically, i.e., $\phi=0$ in $L^{2}\left(\boldsymbol{R}^{n}\right)$.

The purpose of this paper is to provide new criteria for scattering solution to vanish identically.

Our first result is :

Theorem 1. Suppose that a self-adjoint operator $H$ in $\mathscr{H}$ satisfies the following hypotheses (H0) and $(\mathrm{H} 1)_{+}$:

(H0) $\quad H$ has no singular spectrum.

$(\mathrm{H} 1)_{+}$The wave operator

$$
W_{+}=\underset{t \rightarrow+\infty}{\mathrm{S}-\lim _{i}} e^{i t H} e^{-i t H_{0}}
$$

exists and is complete.

Let $\phi \in \mathscr{H}_{\text {cont }}$. If $e^{-\imath t H} \phi$ has the estimate

$$
\left|\left(e^{-i t H} \phi\right)(x)\right| \leqq C t^{-a}(1+|x|)^{-\beta}
$$

for any $t \geqq 1$ and almost all $x \in \mathbb{R}^{n}$, where $\alpha, \beta \in \mathbb{R}$ with $\alpha+\beta>n / 2$ and $C>0$, then $\phi$ vanishes identically.

Note that (0.1) with (0.2) imply (H0) and (H1) + (see, e. g., [5], [14] and [16]). Furthermore, our hypotheses are satisfied even when $V$ has some singularities (see [1] and [14]).

We also remark that if, for simplicity, $\phi \in C_{0}^{\infty}\left(\boldsymbol{R}^{n}\right)$, then

$$
\left|\left(e^{-i t H_{0}} \phi\right)(x)\right| \leqq C|t|^{-\alpha}(1+|x|)^{-\beta} \quad\left(|t| \geqq 1, x \in \mathbb{R}^{n}\right)
$$


holds for any $\alpha, \beta \in R$ with $\alpha+\beta=n / 2$ and $\beta \geqq 0$ (see [4]-[7], [17] and [18]; see also [3] and [16]). It is clear that $H_{0}$ satisfies our assumptions and that $\mathscr{H}_{\text {cont }}\left(H_{0}\right)=L^{2}\left(\mathbb{R}^{n}\right)$.

Therefore, the theorem above clarifies the reason why (0.5) is optimal. (0.5) is one of the essential estimates in the scattering theory, especially in the proof of the existence of wave operators by the Cook-Kuroda method. (Concerning this method, see also [15].)

We shall prove Theorem 1 in $\S 2$. The proof uses isme-dependent method and some simple calculus. This method is based on the lower-bound estimates developed by the author in [13].

Our second result gives lower bounds of decay order in space-time of scattering solutions to the Schrödinger equations :

Theorem 2. Suppose that a self-adjoint operator $H$ in If satısfies (HO) and $(\mathrm{H} 1)_{+}$. Then for any $\phi \in \mathcal{M}_{\text {cont }} \backslash\{0\}, e^{-i t H} \phi$ has the estima:s

$$
\liminf _{R \rightarrow+\infty} R^{-1} \int_{\Gamma_{R}^{+}}\left|\left(e^{-i t H} \phi\right)(x)\right|^{2} d x d t>0,
$$

where $\Gamma_{R}^{+}=\left\{(x, t) \in \mathbb{R}^{n} \times \mathbb{R} ; R<\left(|t|^{2}+|x|^{\mid 2}\right)^{1 / 2}<2 R, t>0\right\}$.

In $\S 3$, we shall prove Theorem 2 by using a theorem of Rellich type due to Hörmander [2] and Murata [10]-[11].

A geometrical observation of the characteristic variety

$$
\xi_{0}+\sum_{j=1}^{n} \xi_{j}^{2}=0 \text { in } \mathbb{R}^{n+1}
$$

will explain the reason why $\alpha+\beta=n / 2$ in (0.5) is critical and why appears the order $\mathcal{O}\left(R^{-1}\right)$ in $(0.6)$.

\section{$\S 1$. Preliminaries}

We recall two theorems in [13]:

Theorem A. Suppose that (H0) and $(\mathrm{H} 1)_{+}$hold. Lct $2 \leqq p \leqq+\infty$ and let $\phi \in \mathscr{H}_{\text {cont. }}$. Assume that there exists $t_{0}>0$ such that

$$
e^{-\imath t H} \phi \in L_{\mathrm{loc}}^{p}\left(\boldsymbol{R}^{n}\right) \quad \text { for all } t>t_{0} .
$$

Then, we have $\phi=0$ if $e^{-i t H} \phi$ satisfies the following condition:

(A) For each $k^{\prime}>k>0$,

$$
\liminf _{t \rightarrow+\infty} t^{n / 2-n / p}\left\|e^{-\imath t H} \phi\right\|_{\left.L^{p}\left(1 k t<|x|<k^{\prime} t\right)\right)}=0
$$


Theorem B. Suppose that (H0) and (H1) + hold. Let $1 \leqq p<2$ and let $\phi \in \mathscr{A}_{\text {cont }}$. Assume that there exists $t_{0}>0$ such that

$$
e^{-i t H} \phi \in L_{\mathrm{loc}}^{\infty}\left(\boldsymbol{R}^{n}\right) \quad \text { for all } t>t_{0} .
$$

Then, we have $\phi=0$ if $e^{-i t H} \phi$ satis.fies either of the following condition (B1)-(B2):

(B1) For each $k^{\prime}>k>0$,

$$
\sup _{t>t_{0}} t^{n / 2}\left\|e^{-i t H} \phi\right\|_{\left.L^{\infty}\left(1 k t<|x|<k^{\prime} t\right)\right)}<+\infty
$$

and

$$
\liminf _{t \rightarrow+\infty} t^{n / 2-n / p}\left\|e^{-i t H} \phi\right\|_{L^{p}\left(\left\{k t<|x|<k^{\prime} t\right)\right.}=0 .
$$

(B2) For each $k^{\prime}>k>0$,

$$
\sup _{t>t_{0}} t^{n / 2-n / p}\left\|e^{-i t H} \phi\right\|_{L^{p}\left(1 k t<|x|<k^{\prime} t\right)}<+\infty
$$

and

$$
\liminf _{t \rightarrow+\infty} t^{n / 2}\left\|e^{-i t H} \phi\right\|_{L^{\infty}\left(\left\{k t<|x|<k^{\prime} t\right\}\right)}=0 .
$$

\section{$\S 2$. Proof of Theorem 1}

Let $\alpha \in \boldsymbol{R}$. It suffices to prove the theorem assuming that $e^{-i t H} \phi$ has the estimate

$$
\left.\left|\left(e^{-i t H} \phi\right)(x)\right| \leqq C t^{-\alpha-\varepsilon}(1+|x|)^{\alpha-n / 2} \quad \text { (for any } t \geqq 1 \text { and a.e. } x\right)
$$

for some $C, \varepsilon>0$.

We shall prove the theorem in the following cases:

1) $\alpha<n / 2$.

2) $\alpha=n / 2$.

3) $\alpha>n / 2$.

1) The case $\alpha<n / 2$.

Set $p=2(n+\varepsilon) /(n-2 \alpha)$. We remark that

$$
p=2+2(2 \alpha+\varepsilon) /(n-2 \alpha)>2
$$

and that

$$
-n-p(\alpha-n / 2)=\varepsilon>0 .
$$

Take $k^{\prime}>k>0$ arbitrary. We have

$$
\begin{aligned}
\left(\int_{k t<|x|<k^{\prime} t}\left|\left(e^{-i t H} \phi\right)(x)\right|^{p} d x\right)^{1 / p} & \leqq C t^{-\alpha-\varepsilon}\left(\int_{k t<|x|<k^{\prime} t}\left(|x|^{\alpha-n / 2}\right)^{p} d x\right)^{1 / p} \\
& =C\left(\omega_{n}(1 / \varepsilon)\left[(1 / k)^{\varepsilon}-\left(1 / k^{\prime}\right)^{\varepsilon}\right]\right)^{1 / p} \cdot t^{-\alpha-\varepsilon-\varepsilon / p}
\end{aligned}
$$

where $\omega_{n}=2 \pi^{n / 2} / \Gamma(n / 2)$. 
Noting that

$$
-\alpha-\varepsilon / p=n / p-n / 2
$$

we obtain

$$
\liminf _{t \rightarrow+\infty} t^{n / 2-n / p}\left\|e^{-i t H} \phi\right\|_{L^{p}\left(1 k t<|x|<k^{\prime} t\right)} \leqq 0 .
$$

From Theorem A, we conclude that $\phi=0$.

2) The case $\alpha=n / 2$.

In this case, we have

$$
\left\|e^{-i t H} \phi\right\|_{L^{\infty}\left(R^{n}\right)} \leqq C t^{-n / 2-\varepsilon} \quad \text { for all } t \geqq 1 .
$$

Thus, (2.6) implies that the condition (A) in Theorem A holds. Consequently, we have $\phi=0$.

3) The case $\alpha>n / 2$.

From (2.1), we see

$$
e^{-i t H} \phi \in L_{\mathrm{loc}}^{\infty}\left(\boldsymbol{R}^{n}\right) \quad \text { for all } t \geqq 1 .
$$

Take $k^{\prime}>k>0$ arbitrary. It follows from (2.1) that

$$
\left\|e^{-i t H} \phi\right\|_{\left.L^{\infty}\left(|k t<| x \mid<k^{\prime} t\right)\right\}} \leqq C t^{-\alpha-\varepsilon}\left(1+k^{\prime} t\right)^{\alpha-n / 2} \quad \text { for all } t \geqq 1 .
$$

On the other hand, we find

$$
\left\|e^{-i t H} \phi\right\|_{\left.L^{1}\left(1 k t<\mid x<k^{\prime} t\right)\right)} \leqq C \omega_{n} k^{\prime n / 2+a} t^{n / 2-\varepsilon} /(n / 2+\alpha) \quad \text { for all } t \geqq 1 .
$$

Hence, $e^{-i t H} \phi$ satisfies both (B1) and (B2). In view of Theorem B, we obtain $\phi=0$.

This completes the proof.

Remark 1. For the case of $t<0$, we have an analogous theorem as follows:

Theorem. Suppose that a self-adjoint operator $H$ in $\mathscr{A}$ satisfies (H0) and (H1)_:

(H1)_ The wave operator

$$
W_{-}=\mathrm{s}_{t \rightarrow-\infty} \lim ^{i t H} e^{-i t H_{0}}
$$

exists and is complete.

Let $\phi \in \mathscr{H}_{\text {cont. }}$ If $e^{-i t H} \phi$ has the estimate

$$
\left|\left(e^{-i t H} \phi\right)(x)\right| \leqq C|t|^{-a}(1+|x|)^{-\beta}
$$

for any $|t| \geqq 1$ and almost all $x \in \mathbb{R}^{n}$ where $\alpha, \beta \in \mathbb{R}$ with $\alpha+\beta>n / 2$ and $C>0$, then $\phi$ vanishes identically. 


\section{$\S$ 3. Lower Bounds of Decay Order in Space-Time for Scattering Solutions to the Schrödinger Equations (Proof of Theorem 2)}

We consider a partial differential operator $P(-i \partial)$ in $\mathbb{R}^{m}(m \geqq 1)$ with constant coefficients.

Hörmander [2] and Murata [10]-[11] proved :

Assume that $V(P):=\left\{\xi \in \mathbb{R}^{m} ; P(\xi)=0\right\}$ is non-empty and of codimension $k$. Let $\Gamma$ be an open cone in $\boldsymbol{R}^{m}$ such that for every real analytic manifold $M \subset V(P)$ and for every $x^{0} \in M, \Gamma$ contains some normal of $M$ at $x^{0}$. If $u \in \mathscr{S}^{\prime}\left(\boldsymbol{R}^{m}\right) \cap$ $L_{\text {loc }}^{2}\left(\boldsymbol{R}^{m}\right)$ satisfies

$$
P(-i \partial) u=0
$$

and

$$
\liminf _{R \rightarrow+\infty} R^{-k} \int_{\Gamma_{R}}|u(x)|^{2} d x=0,
$$

where $\Gamma_{R}=\{x \in \Gamma ; R<|x|<2 R\}$, then $u=0$.

Note that

$$
P\left(\xi_{0}, \xi_{1}, \cdots, \xi_{n}\right):=\xi_{0}+\sum_{j=1}^{n} \xi_{j}^{2}, \quad\left(\xi_{0}, \cdots, \xi_{n}\right) \in \mathbb{R}^{n+1},
$$

with $m=n+1, k=1$ and

$$
\Gamma:=\left\{x \in \boldsymbol{R}^{n+1} ; x_{0}>0\right\} \quad \text { or } \quad\left\{x \in \boldsymbol{R}^{n+1} ; x_{0}<0\right\}
$$

satisfies the assumption of the theorem above. Therefore, if $\phi \in L^{2}\left(\boldsymbol{R}^{n}\right)$ satisfies

$$
\liminf _{R \rightarrow+\infty} R^{-1} \int_{\Gamma_{R}^{ \pm}}\left|\left(e^{-i t H_{0}} \phi\right)(x)\right|^{2} d x d t=0,
$$

then $\phi=0$.

We are now in a position to prove Theorem 2 .

Proof of Theorem 2. Let $\phi \in \mathscr{M}_{\text {cont }} \backslash\{0\}$. From the assumptions, we have that $\phi \in$ Range $\left(W_{+}\right), W_{+}^{*} \phi \neq 0$ and that

$$
\lim _{t \rightarrow+\infty}\left\|e^{-i t H} \phi-e^{-i t H_{0}} W_{+}^{*} \phi\right\|=0,
$$

where $W_{+}^{*}$ denotes the adjoint of $W_{+}$. Noting that

$$
\sup _{t \in R}\left\|e^{-i t H} \phi-e^{-i t H_{0}} W_{+}^{*} \phi\right\|^{2} \leqq 4\|\phi\|^{2} .
$$

we easily obtain

$$
\lim _{R \rightarrow+\infty} R^{-1} \int_{0}^{2 R}\left\|e^{-i t H} \phi-e^{-i t H_{0}} W_{+}^{*} \phi\right\|^{2} d t=0 .
$$


The preceding observation implies

$$
\liminf _{R \rightarrow+\infty} R^{-1} \int_{\Gamma_{R}^{+}}\left|\left(e^{-\imath t H_{0}} W^{*} \phi\right)(x)\right|^{2} d x d t>0 .
$$

From the following elementary relation in a normed space $E$ :

$$
\|u\|^{2} \geqq \frac{1}{2}\|v\|^{2}-\|u-v\|^{2}, \quad u, v \in E,
$$

it follows that

$$
\begin{aligned}
& R^{-1} \int_{\Gamma_{R}^{+}}\left|\left(e^{-\imath t H} \phi\right)(x)\right|^{2} d x d t \\
\geqq & (2 R)^{-1} \int_{\Gamma_{R}^{+}}\left|\left(e^{-\imath t H_{0}} W_{+}^{*} \phi\right)(x)\right|^{2} d x d t-R^{-1} \int_{\Gamma_{R}^{+}}\left|\left(e^{-\imath t H} \phi-e^{-\imath t H_{0}} W_{+}^{*} \phi\right)(x)\right|^{2} d x d t .
\end{aligned}
$$

The second term of the right side of (3.7) is estimated as follows:

$$
\begin{aligned}
& -R^{-1} \int_{\Gamma_{R}^{+}}\left|\left(e^{-\imath t H} \phi-e^{-\imath t H_{0}} W_{+}^{*} \phi\right)(x)\right|^{2} d x d t \\
\geqq & -R^{-1} \int_{0}^{2 R}\left\|e^{-\imath t H} \phi-e^{-\imath t H_{0}} W_{+}^{*} \phi\right\|^{2} d t .
\end{aligned}
$$

Combining (3.5)-(3.8), we conclude that

$$
\liminf _{R \rightarrow+\infty} R^{-1} \int_{\Gamma_{R}^{+}}\left|\left(e^{-\imath t H} \phi\right)(x)\right|^{2} d x d t>0 .
$$

This completes the proof.

Remark 2. If we assume (H0) and (H1)_, we have an analogous result for the case of $\Gamma_{\vec{R}}$.

Remark 3. The proof of Theorem 2 can be applied to the case of nonlinear Schrödinger equation with a single power interaction:

$$
\begin{aligned}
& i \partial_{t} u=-\Delta u+|u|^{p-1} u, \quad \text { on } \quad(x, t) \in \mathbb{R}^{n} \times \mathbb{R} \quad(n \geqq 1), \\
& u(x, 0)=\phi(x), \quad x \in \mathbb{R}^{n},
\end{aligned}
$$

where $1+2 / n<p<\alpha(n)$ with $\alpha(n):=+\infty(n=1,2)$ and $\alpha(n):=(n+2) /(n-2)$ $(n \geqq 3)$. We easily obtain :

For any $\dot{\phi} \in \mathscr{H} \backslash\{0\}$ with $\nabla \phi, x \phi \in \mathscr{H}$, the unique mild solution $u$ of (NLS) has the estimate

$$
\liminf _{R \rightarrow+\infty} R^{-1} \int_{\Gamma_{R}^{ \pm}}|u(x, t)|^{2} d x d t>0 .
$$

See also [13] for lower bounds of $L^{p}$-decay in time for solutions to (NLS). 


\section{References}

[1] Davies, E. B., On Enss' approach to scattering theory, Duke Math. J., 47 (1980), 171-185.

[2] Hörmander, L., Lower bounds at infinity for solutions of differential equations with constant coefficients, Israel J. Math., 16 (1973), 103-116.

[3] - The existence of wave operators in scattering theory, Math. Z., 146 (1976), 69-91.

[4] Jörgens, K. and Weidmann, J., Zur existenz der Wellenoperatoren, Math. Z., 131 (1973), 141-151.

[5] Kato, T. and Kuroda, S. T., The abstract theory of scattering, Rocky Mountain Journal of Mathematics, 1 (1971), 127-171.

[6] Kupsh, J. and Sandhas, W., M $\phi 1$ ler Operators for scattering on singular potentials, Comm. Math. Phys., 2 (1966), 147-154.

[7] Kuroda, S.T., On the existence and unitary property of the scattering operator, Nuovo Cimento, 12 (1959), 431-454.

[8] Masuda, K., On exponential decay of solutions for some partial differential equations, J. Math. Soc. Japan., 19 (1967), 82-90.

[9] — Asymptotic behavior in time of solutions for evolution equations, $J$. Funct. Anal., 1 (1969), 84-92.

[10] Murata, M., A theorem of Liouville type for partial differential equations with constant coefficients, J. Fac. Sci. Univ. Tokyo, 21 (1974), 395-404.

[11] —, Asymptotic behaviors at infinity of solutions of certain linear partial differential equations, J. Fac. Sci. Univ. Tokyo, 23 (1976), 107-148.

[12] — Rate of decay of local energy and spectral properties of elliptic operators, Japan. J. Math., 6 (1980), 77-127.

[13] Ozawa, T., Lower $L^{p}$-bounds for scattering solutions of the Schrödinger equations, Preprint, (1986).

[14] Perry, P., Scattering theory by the Enss method, Mathematical Reports, 1 (1983), $1-347$.

[15] Schechter, M., A new criterion for scattering theory, Duke Math. J., 44 (1977), 863-872.

[16] Simon, B., Phase space analysis of simple scattering systems: extensions of some work of Enss, Duke Math. J., 46 (1979), 119-168.

[17] Veselić, K. and Weidmann, J., Existenz der wellenoperatoren für eine allgemeine klasse von operatoren, Math. Z., 134 (1973), 255-274.

[18] - Asymptotic estimates of wave functions and the existence of wave operators, J. Funct. Anal., 16 (1974), 61-77. 